\title{
XIST-induced silencing of flanking genes is achieved by additive action of repeat a monomers in human somatic cells
}

\author{
Jakub Minks, Sarah EL Baldry, Christine Yang, Allison M Cotton and Carolyn J Brown*
}

\begin{abstract}
Background: The establishment of facultative heterochromatin by X-chromosome inactivation requires the long non-coding RNA XIST/Xist. However, the molecular mechanism by which the RNA achieves chromosome-wide gene silencing remains unknown. Mouse Xist has been shown to have redundant domains for cis-localization, and requires a series of well-conserved tandem ' $A$ ' repeats for silencing. We previously described a human inducible XIST transgene that is capable of cis-localization and suppressing a downstream reporter gene in somatic cells, and have now leveraged these cells to dissect the sequences critical for XIST-dependent gene silencing in humans.

Results: We demonstrated that expression of the inducible full-length XIST CDNA was able to suppress expression of two nearby reporter genes as well as endogenous genes up to $3 \mathrm{MB}$ from the integration site. An inducible construct containing the repeat A region of XIST alone could silence the flanking reporter genes but not the more distal endogenous genes. Reporter gene silencing could also be accomplished by a synthetic construct consisting of nine copies of a consensus repeat A sequence, consistent with previous studies in mice. Progressively shorter constructs showed a linear relationship between the repeat number and the silencing capacity of the RNA. Constructs containing only two repeat A units were still able to partially silence the reporter genes and could thus be used for site-directed mutagenesis to demonstrate that sequences within the two palindromic cores of the repeat are essential for silencing, and that it is likely the first palindrome sequence folds to form a hairpin, consistent with compensatory mutations observed in eutherian sequences.

Conclusions: Silencing of adjacent reporter genes can be effected by as little as 94 bp of XIST, including two 'monomers' of the A repeat. This region includes a pair of essential palindromic sequences that are evolutionarily well-conserved and the first of these is likely to form an intra-repeat hairpin structure. Additional sequences are required for the spread of silencing to endogenous genes on the chromosome.
\end{abstract}

Keywords: X-chromosome inactivation, XIST, Long non-coding RNA, Eutherian dosage compensation, Gene silencing

\section{Background}

To ensure dosage compensation of X-linked genes between males and females, eutherian females silence one $\mathrm{X}$ chromosome [1]. The minimal region required for $\mathrm{X}$ chromosome inactivation contains the non-coding (nc) RNA gene XIST, which is expressed solely from the inactive $\mathrm{X}$ chromosome [2]. Experiments in mice have shown that Xist is both required and sufficient for

\footnotetext{
*Correspondence: carolyn.brown@ubc.ca

Department of Medical Genetics, Molecular Epigenetics Group, University of British Columbia, Life Sciences Institute, 2350 Health Sciences Mall, Vancouver, BC V6T 1Z3, Canada
}

inactivation; however, the mechanism by which the XIST/Xist RNA causes chromosome-wide gene silencing remains elusive (reviewed in [3]). XIST localizes in cis to the chromatin of the inactive $\mathrm{X}$ chromosome [4], suggesting a potential role in targeting silencing complexes to the chromosome. The alternatively spliced and polyadenylated RNA is over $15 \mathrm{~kb}$ long in all eutheria examined. Overall the gene is only weakly conserved among mammals, but its regions of repetitive sequences called repeat A to F show better conservation $[4,5]$. Additionally, exon 4 of XIST/Xist is well-conserved, and

\section{Biomed Central}


shows homology with the protein-coding $\operatorname{Ln} x 3$ gene, from which the Xist gene may have evolved by the addition of sequences from transposable elements [6,7]. Intriguingly, in marsupials, $L n x 3$ remains protein-coding and Rsx3 encodes an RNA that is similar to XIST in that the long non-coding, repeat-rich RNA is transcribed from and associates with the inactive $\mathrm{X}$ chromosome [8]. While there is no sequence conservation between Rsx3 and XIST, both are able to silence in cis, and show regions of putative stem-loop structure, supporting the idea that these long ncRNAs may be serving as adapter molecules containing different protein-recognition motifs to recruit components of the gene-silencing machinery to the inactive $\mathrm{X}$ chromosome.

As X-chromosome inactivation is a developmental process, most studies of Xist function have been undertaken in mice, where embryonic stem (ES) cells or embryos can be analyzed during the inactivation process. Human ES cells have demonstrated considerable epigenetic instability (for example, [9]) and studies of human embryos are necessarily restricted $([10,11])$. However, the potential differences in the inactivation process between mice and humans, suggested by both differences in the regulation of the XIST gene and the number of genes escaping inactivation (reviewed in [12]), led us to develop an inducible model to study human XIST action [13]. Induced expression of XIST in the immortal HT1080 fibrosarcoma cell line is able to induce some features of an inactive X, including XIST localization, silencing of a co-integrated reporter gene, depletion of repetitive (CoT1) RNA, and the acquisition of some heterochromatic histone modifications associated with the inactive $\mathrm{X}$.

Previous studies in mice targeted a panel of truncated inducible Xist transgenes to the single X chromosome in a male ES cell and demonstrated that redundant sequences were involved in localization of the mouse Xist RNA to the chromosome [14], with a construct containing only approximately $3 \mathrm{~kb}$ of Xist cDNA, including the wellconserved A repeat region, able to localize to and repress the single $\mathrm{X}$ chromosome. Furthermore, chromosomal silencing was fully compromised when the 5' region encompassing repeat A was deleted [14], but concatamers of a synthetic version of these repeats were able to replace the A repeat region. A near normal complement of 7.5 repeats or an increase to 12 repeats fully recapitulated silencing, while 5.5 repeats showed less silencing and 4 repeats were only minimally active [14]. Therefore, in mice the A repeats are necessary for silencing, but additional redundant domains of Xist are involved in localization to the chromosome and the presence of different domains supports models that the RNA is serving as an adaptor to bring different epigenetic silencing proteins to the inactive $\mathrm{X}$.
A number of chromatin remodeling proteins associate with the inactive $\mathrm{X}$ chromosome resulting in the acquisition of many histone modifications characteristic of heterochromatin (reviewed in [3]). The binding of many of these proteins is Xist-dependent; and it has been shown that the A repeat region interacts in vitro and in vivo with components of PRC2 [15-17]. Surprisingly, however, a silencing defective Xist RNA that lacks the repeat A region is still able to recruit PRC2, PRC1, SAF-A, ASH2L and macroH2A1 to the inactive $\mathrm{X}$ chromosome in ES cells (reviewed in [3]). In contrast, a similar deletion in transgenic mice failed to produce Xist RNA, suggesting an important regulatory role for the repeat A region [18]. Furthermore, interaction with the transcriptional repressor YY1 [19] has been shown to occur at the mouse $C$ repeat region and while a direct interaction with the A repeat region has been reported for the splicing factor ASF/SF2, this has been proposed to have a role in enabling proper processing of the Xist RNA facilitating choice of the future inactive $\mathrm{X}$ chromosome [20]. Therefore, despite the growing body of literature on XIST/Xist-interacting partners and identification of a critical role for the A repeat region, understanding how XIST/Xist expression leads to gene silencing remains elusive. Contributing to the challenge is the large size of the XIST RNA, and that the monitoring of silencing at distal sites requires both silencing and spread of the RNA along the chromosome.

The palindromic nature of the repeat A core sequences suggests their involvement in forming a distinct secondary RNA structure, and several alternative but mutually exclusive structures have been suggested. The first model proposed that each of the two palindromes forms a hairpin and thus the repeat A region of XIST RNA folds into a two-hairpin 8- or 9-mer [14]. This structure was supported by the abrogation of silencing activity in a construct with two base alterations that would disrupt the putative first hairpin. However, an in vitro analysis of repeat A structure by fluorescence resonance energy transfer, as well as sensitivity to RNases that specifically digest single- or double-stranded RNA regions, proposed an alternative structure. The first palindrome was suggested to engage in pairing between two separate monomers, rather than within repeat $\mathrm{A}$ monomers, and the model proposed that the second palindrome did not form a defined structure [16]. Recently, a third option, supported by nuclear magnetic resonance analyses of repeat A monomer and dimer structures, suggested that under in vitro conditions, the first palindrome forms a hairpin, while the second palindrome engages in pairing between repeat A units [21,22].

Our previously reported inducible transgenic system in the immortal fibrosarcoma line HT1080 provides a tractable system to study the RNA sequences involved in gene 
repression by XIST [13]. Here, we focus on refining the minimal XIST sequence necessary for cis-regulated silencing, independent of the developmental signals that establish mono-allelic XIST expression in females. We demonstrate silencing of reporter genes by expression of less than $100 \mathrm{bp}$ of XIST containing two consensus repeat A monomers.

\section{Results and discussion}

Repeat $A$ is sufficient for XIST-dependent reporter gene silencing

We have previously shown that an inducible transgenic $X I S T$ is capable of silencing an Enhanced Green Fluorescent
Protein gene (EGFP) reporter in human somatic cells, while a construct lacking the repeat $A$ region failed to silence the EGFP gene [13]. Similarly, inducible mouse constructs have been shown to require the repeat $\mathrm{A}$ region for silencing of the X chromosome in mouse ES cells [14]. The RNA induced from the full-length XIST cDNA construct localizes to the autosome upon which it has integrated [13]; however, the EGFP reporter construct is located only $7.7 \mathrm{~kb} 3$ ' of XIST in HT1080 male fibrosarcoma cells (see Figure 1A) and, thus, may not require the localization domains of XIST for silencing. Therefore, to test whether the repeat A is sufficient for proximal gene silencing, we induced expression

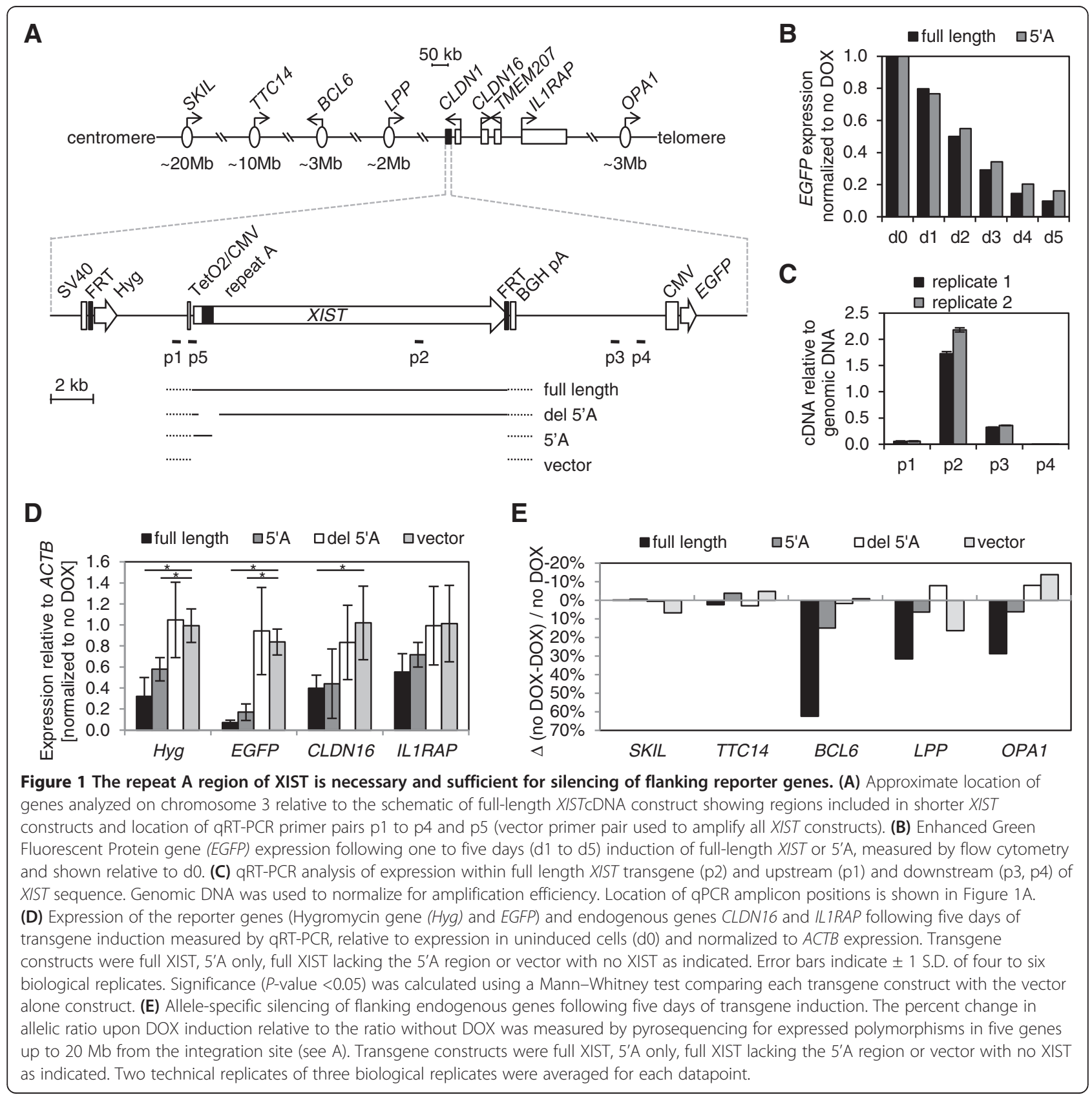


of a construct containing only repeat A sequence (5'A) and measured EGFP expression by flow cytometry (Figure 1B). The extent and dynamics of EGFP silencing by repeat A mimicked that of the full XIST construct over five days following induction of the construct's expression by doxycycline (DOX), suggesting that the ability of XIST to silence the proximal EGFP reporter gene is attributable to the repeat A region.

To confirm that silencing results from an XIST RNArelated, sequence-specific effect rather than transcriptional interference, we demonstrated that transcription of the DOX-induced XIST transgenes ceased before the reporter construct. While some transcripts were present downstream of the polyadenylation site, transcription was completely absent at a site approximately $2 \mathrm{~kb} 5$ ' of the EGFP promoter (Figure 1C). Our conclusion that silencing is not due to transcriptional interference is further supported by XIST-dependent attenuation of the expression of the hygromycin resistance gene $(\mathrm{Hyg})$ located upstream of XIST and absence of gene silencing with vector lacking XIST sequences (Figure 1D).

\section{Endogenous gene silencing induced by full-length XIST}

In order to explore whether XIST RNA is able to induce silencing of the endogenous genes flanking the integration site, we identified the FRT integration site into which subsequent single-copy integrations were directed. DNA-FISH using the XIST cDNA identified the full-length transgene as integrated onto the $\operatorname{der}(11) \mathrm{t}(3 ; 11)$ of $46, \mathrm{XY}$,del (1)(p21),i(3)(p10),i(3)(q10),der(4)t(1;4)(p21;p16), der(5)t(5;5) (p15;?),der(11)t(3;11)(q11;q25) cells. We used inverse PCR from primers in the pFRT/lacZeo plasmid to identify the 3q FRT integration site as just downstream of the CLDN1 gene (Figure 1A). Low expression levels of the CLDN1, TMEM207 and LEPREL1 genes prevented a reliable analysis of these adjacent genes by qRT-PCR. Using qRT-PCR following induction of full-length XIST, we observed significant silencing of CLDN16, a gene located approximately $100 \mathrm{~kb}$ downstream of XIST (Figure 1D). Neither the construct consisting only of repeat A, nor the construct containing a deletion of repeat A showed significant silencing of CLDN16 upon induction, although there was a non-significant reduction with the repeat A-containing construct. IL1RAP, which is located a further $120 \mathrm{~kb}$ downstream (that is, $220 \mathrm{~kb}$ from XIST) did not show significant XIST-induced silencing, although there was a non-significant drop in expression. The decrease in CLDN16 transcription is consistent with the almost complete silencing of the cis-located allele; however, attempts to confirm silencing of the XIST-associated allele by FISH failed, presumably due to the relatively low expression levels of CLDN16. In order to examine whether one allele of endogenous genes was being silenced, we identified more distal genes that contained an expressed polymorphism, and thus provided an opportunity to probe allelic silencing. At the DNA level these genes show an allelic ratio of approximately 66\%, consistent with the presence of a single allele on the $\operatorname{der}(11) t(3 ; 11)$ and the alternate allele in two copies on the isochromosome 3q. Upon DOX treatment there was a significant decrease in relative expression of the single allele for BCL6, LPP and OPA1 (Figure 1D), shown as a change upon DOX induction, relative to the expression in cells without DOX treatment, as there can be variations in allelic expression levels. Similar to the q-PCR results with CLDN16, constructs containing $X I S T$ lacking repeat $\mathrm{A}$, or no XIST (vector sequences only) showed no change in allelic ratio upon DOX induction; however, in these cell lines the DNA ratio showed an equivalent allelic DNA ratio, reflecting karyotypic instability of the HT1080 line. There was a significant allelic silencing of BCL6 with the construct containing only repeat $\mathrm{A}$; however, the reduced silencing seen with this construct suggests that additional sequences are required for the spread of the XISTinduced silencing effect beyond the immediate XIST domain.

As repeat A binds the polycomb group 2 proteins that are responsible for trimethylation of $\mathrm{H} 3 \mathrm{~K} 27$, we asked whether there would be a differential ability of fulllength versus repeat A alone to recruit H3K27me3. However, we did not observe any H3K27me3 enrichment by ChIP at the EGFP, Hyg or the CLDN16 promoters (Additional file 1: Figure S1). H3K27me3 is a mark of the inactive $X$, and has been shown to be enriched at the promoters of inactivated genes [23]; however, given that the silencing we have observed in this system is reversible ([13] and data not shown), it is perhaps not surprising that this heritable mark of silent chromatin is not recruited. Similarly, we had previously shown that there was no recruitment of DNA methylation in this reversible system [13]. A similar inducible transgene in mice had identified a developmental window during which inactivation could occur [24], yet we observe induction of silencing in our somatic cell model; possibly reflecting a more epigenetically dynamic state to these cancer-derived cells, or differences in the genes being examined, as we observed variability between genes in their ability to be silenced. By recapitulating XIST-induced gene silencing, but not requiring sequences involved in the spread of XIST, the A repeat construct exposes the most basal aspects of XIST silencing function. To identify the minimal functional unit for silencing, we further dissected the repeat $\mathrm{A}$ sequences.

\section{Repeat A monomers contribute additively to silencing}

In order to further characterize the link between repeat A sequence and its silencing ability, we generated an 
artificial repeat A construct that tested the potential impact of sequence variations in the individual monomers, which are particularly prevalent in the T-rich linker regions. This artificial repeat $\mathrm{A}$ consisted of a nine-fold repetition of a 46 bp consensus monomer sequence, and contained restriction enzyme sites in the T-rich stretches to allow for the creation of constructs with reduced numbers of repeats (Figure 2A). Flow cytometry and qPCR showed that the artificial repeat A silenced EGFP to the same extent as full-length XIST or human repeat
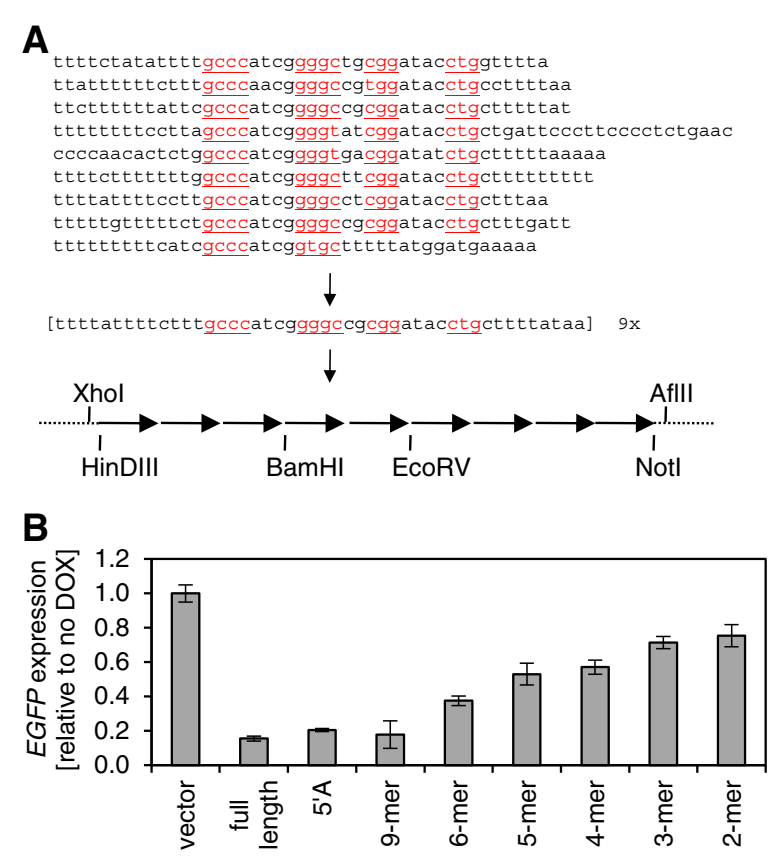

C

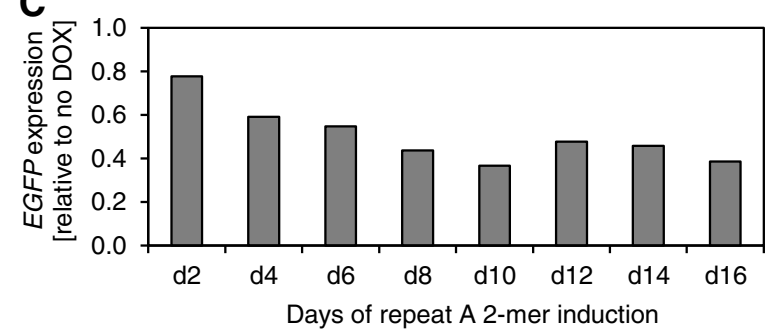

Figure 2 Repeat A monomers additively contribute to

silencing. (A) Human repeat A sequence consists of 8.5 copies of a well-conserved CG-rich core and T-rich spacer sequences.

Palindromic sequences hypothesized to form a secondary structure are underlined. Artificial repeat A was constructed as a 9-mer

repetition of consensus monomer sequence and restriction enzyme sites were introduced to allow for the creation of shorter constructs. (B) Enhanced Green Fluorescent Protein gene (EGFP) expression following five days of transgene induction as measured by GRT-PCR, relative to $\mathrm{d} 0$ and normalized to changes in expression caused by induction of the vector alone and to ACTB expression for two biological replicates. (C) EGFP expression was measured by flow cytometry every 2 days for 16 days following induction of repeat $A$ 2-mer. Data are normalized to EGFP expression in cells that were not induced with DOX.
A constructs. Since variability within the individual repeats and spacer regions did not contribute to silencing, we were then able to test the silencing ability of constructs with fewer repeats. Transgenes harboring two to six repeat A monomers were functional, with an approximately linear relationship between the number of repeats and their silencing ability (Figure 2B). Silencing induced by the repeat A 2-mer gradually increased between day 2 and approximately day 8; however, longer induction of the repeat A 2-mer did not promote further EGFP down-regulation (Figure 2C).

These observations provide strong evidence that the silencing of an adjacent EGFP reporter is achieved through an additive effect of repeat A monomers, with even a 2-mer repeat A inducing partial EGFP silencing. The number of repeat A units was previously reported to correlate with the ability of Xist to induce silencing in differentiating mouse ES cells [14]. Also, in agreement with a previous report on mouse Xist [14], artificial repeat A retains full silencing potential when compared to human repeat $A$, suggesting that neither sequence variation within the CG-rich core nor the varying length of the T-rich spacers in individual repeat A monomers is essential for XIST function. The remarkable ability of a construct with only two repeats to silence EGFP in a reproducible and statistically significant fashion provided us with a well-defined template for further dissection of the relationship between repeat $\mathrm{A}$ sequence and its silencing ability.

The core repeat A sequence consists of two palindromes; the first potentially allowing for perfect C-G pairing linked by 'ATCG' and the second involving C-G pairing as well as a G-U pair linked by 'ATAC' with the T-rich stretches serving as spacers [14]. While alternative structures have since been proposed, for simplicity, we refer to the four components of CG-rich consensus core as stem 1 (S1), loop 1 (L1), stem 2 (S2) and loop 2 (L2). We initially created a variant of the 2-mer repeat A in each of these elements in order to probe their role in cis-silencing of EGFP (Figure 3A). Mutations of L1, S2 and L2 completely ablated the transgenes' ability to silence EGFP, as measured by flow cytometry of two representative clones for each mutation, compared to a canonical repeat A 2-mer (Figure 3B). Analysis by qRTPCR showed the same trends and allowed examination of the Hyg gene (Figure 3C); however, flow cytometry affords considerably greater sensitivity as 30,000 events were combined into each datapoint. Mutation of S1 resulted in partial abolition of EGFP silencing. Thus, the most conserved regions of XIST both among the individual repeats in human (Figure 2A) and among different species (Additional file 2: Figure S2), the CG-rich palindromes and their intervening 'ATCG' and 'ATAC' sequences, are critical for XIST function. All of the 


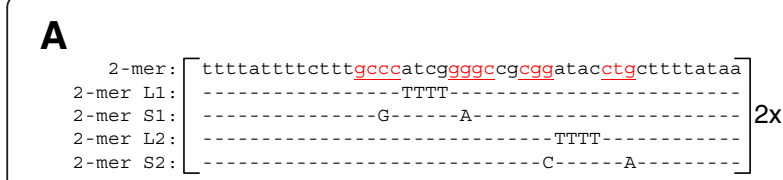

B
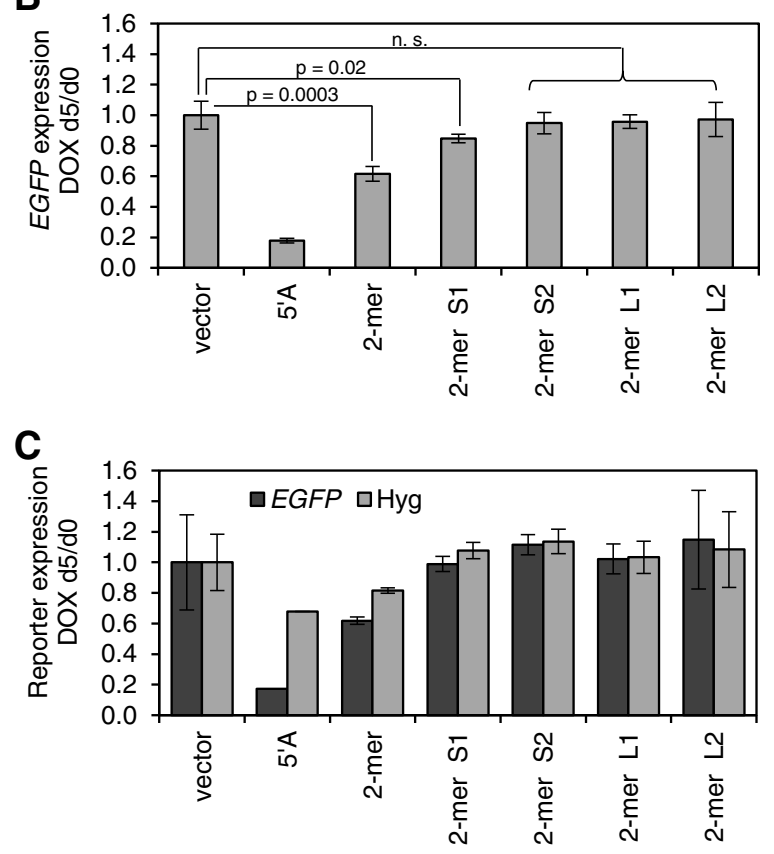

Figure 3 Mutation of the core repeat $A$ sequences abrogates its silencing ability. (A) Sequence of the canonical repeat A monomer and four mutant constructs that were created to target the hypothesized repeat A hairpins. Underlined sequences correspond to stem 1 and stem 2. Dashes indicate no sequence change. (B) Mean Enhanced Green Fluorescent Protein gene (EGFP) expression following five days of transgene induction, measured by flow cytometry, relative to d0 (two-tailed paired t-test). Error bars indicate \pm 1 S.D. of two single-cell clones. (C) EGFP and hygromycin $(\mathrm{Hyg})$ gene expression following five days of transgene induction, measured by qRT-PCR, relative to d0 and normalized to ACTB expression for two independent single-cell clones.

previously proposed structures predict the existence of an 'ATCG' loop and mutation to 'TTTT' in our system completely abolished human repeat A function. Similarly, mutation to 'TAGC' in mice has been shown to partially abolish Xist function [14], suggesting that the sequence of the tetraloop, and not just its presence, is critical for XIST/Xist function.

The palindromic nature of the repeat A core sequences strongly suggests their involvement in forming a distinct secondary RNA structure. Several alternative but mutually exclusive structures were previously proposed in which the CG-rich palindrome encompassing the 'ATCG' tetraloop ('stem 1') may either form a hairpin with pairing within each repeat A monomer [14,21,22] or pairing between two separate monomers [16]. The ability of the repeat A 2-mer to reproducibly induce gene silencing allowed us to use mfold, an RNA structure prediction algorithm [25], to design repeat A mutants that would compare the silencing effectiveness when inter- or intra-repeat pairing was enforced. We found that modeling mutated constructs of larger than 2-mer repeat A structures was highly unreliable as multiple structures of similar minimum free energies (dG) were predicted. We designed a quartet of mutations in the 2-mer repeat $A$ that were predicted to enforce pairing either within (A1, A2) or between (B1, B2) each monomer (Figure 4A and Additional file 3: Figure S3). The repeat A 2-mer mutations were constructed so that a single prominent structure was predicted to fold with either higher (A1, B1), or lower (A2, B2) dG, compared to the unmodified repeat A 2-mer.

Measured by flow cytometry, the mutants predicted to enforce pairing within each monomer functioned better than those enforcing the interaction between the monomers; although none of the four mutants silenced EGFP as efficiently as the canonical repeat A 2-mer (Figure 4B), suggesting that there may be more complex structures involved. While the differences in EGFP expression were relatively subtle due to a limited silencing effect of the repeat A 2-mer, they were highly statistically significant and equivalent results were obtained for two single-cell clones of independent integrations and a total of seven biological replicates. More representative structures containing greater than two repeat units were not tested as they could not be predicted to reliably form only a single thermodynamically favored structure. However, given the number of eutherian genome sequences that have now been assembled, we turned instead to a characterization of the full repeat A sequences that are available in genome databases.

\section{Survey of repeat A mutations shows strong preference for stem 1 and mild preference for stem 2 formation} Taking advantage of the increasing number of sequenced mammalian genomes, we generated an alignment of repeat A sequences from 27 mammalian species (Additional file 2: Figure S2A). Repeat A consists of 24 bp-long CGrich core sequences separated by approximately 20 to 50 bp-long T-rich spacers. The CG-rich core is formed by two palindromes, each of which is broken by four bp-long sequences. As expected, repeat A was well conserved, in particular within the CG-rich core sequences (Additional file 2: Figure S2B). Interestingly, 22/27 mammalian XIST sequences contained either eight or nine monomers of repeat $\mathrm{A}$, and at least one of the remaining five was incomplete across the region, supporting the need for eight monomers to achieve full XIST functionality.

Of the defined stem-loop structures, loop 1 showed the highest frequency of deviation from the canonical 'ATCG' sequence, with approximately 10\% (20/202) of 

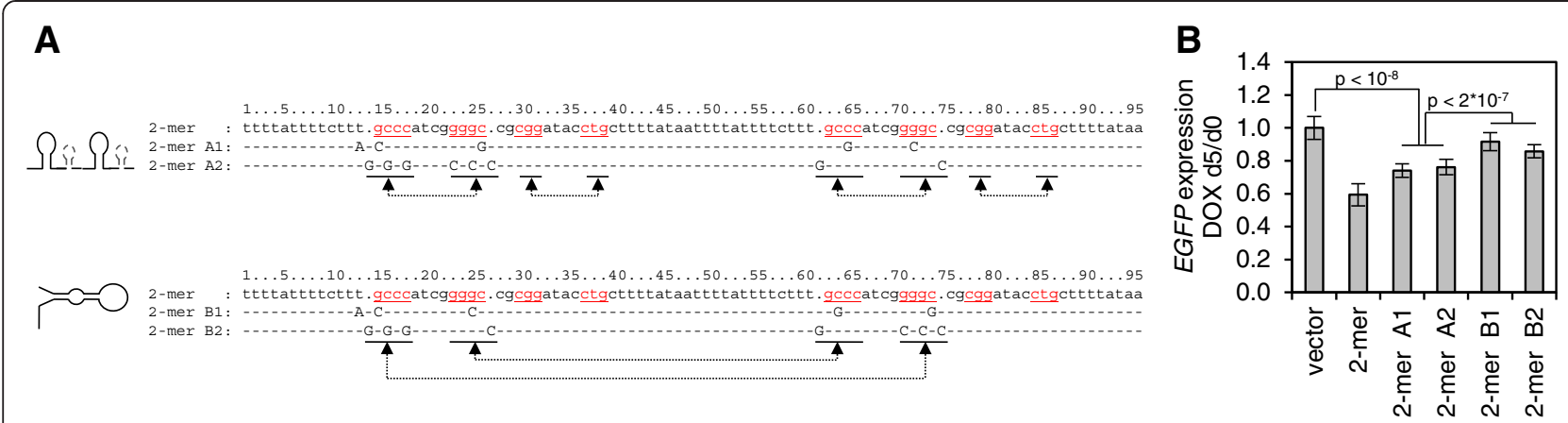

Figure 4 Stem-loop 1 structure is required to maintain repeat A silencing ability. Silencing ability of 2-mer repeat A construct is retained when forced to form stem-loop 1 structure, but abrogated when pairing between the monomers is enforced. (A) Sequence of the canonical repeat A 2-mer and four mutant constructs that either enforce formation of stem-loop 1 (A1, A2) or an alternative folding (B1, B2) of repeat A sequences, as indicated by schematics. Dashes indicate no change in sequence. (B) Mean Enhanced Green Fluorescent Protein gene (EGFP) expression following five days of transgene induction, measured by flow cytometry, relative to d0 (two-tailed paired $t$-test). Error bars indicate \pm 1 S.D. of two independent single-cell clones and a total of seven biological replicates.

repeat A units harboring an 'AACG' tetraloop instead (Additional file 2: Figure S2). To ask whether there was an evolutionary preference for reciprocal mutations that supported the formation of an intra- or interloop configuration we examined deviations from the canonical stem sequences across the bona fide monomers of the 27 mammals (Figure 5 and Additional file 4: Figure S4). Despite the strong conservation there were 50 stem 1 changes, allowing us to determine whether fully complementary double-stranded sequences could form due to existing reciprocal mutations either within the same unit, or in another unit of the same species. Of the 50 stem 1 mutations we analyzed, 24 could not be linked with a reciprocal mutation; 12 of the remaining $26 \mathrm{mu}-$ tations were accompanied by a reciprocal mutation exclusively within the same unit; and a further 10 could pair either within the same unit, or with another unit (Figure 5A). These findings strongly argue in favor of the predicted stem-loop 1 formation. Survey of stem 2 mutations uncovered 46 deviating repeat A units, 28 of which could not pair with any reciprocal mutation (Figure 5B). Of the remaining 18 mutations, 8 could exclusively form a stem-loop by pairing within each unit, with a further 3 allowing for pairing either within a unit or with other units (Figure 5B). While the propensity of the stem 2 region to harbor reciprocal mutations retaining
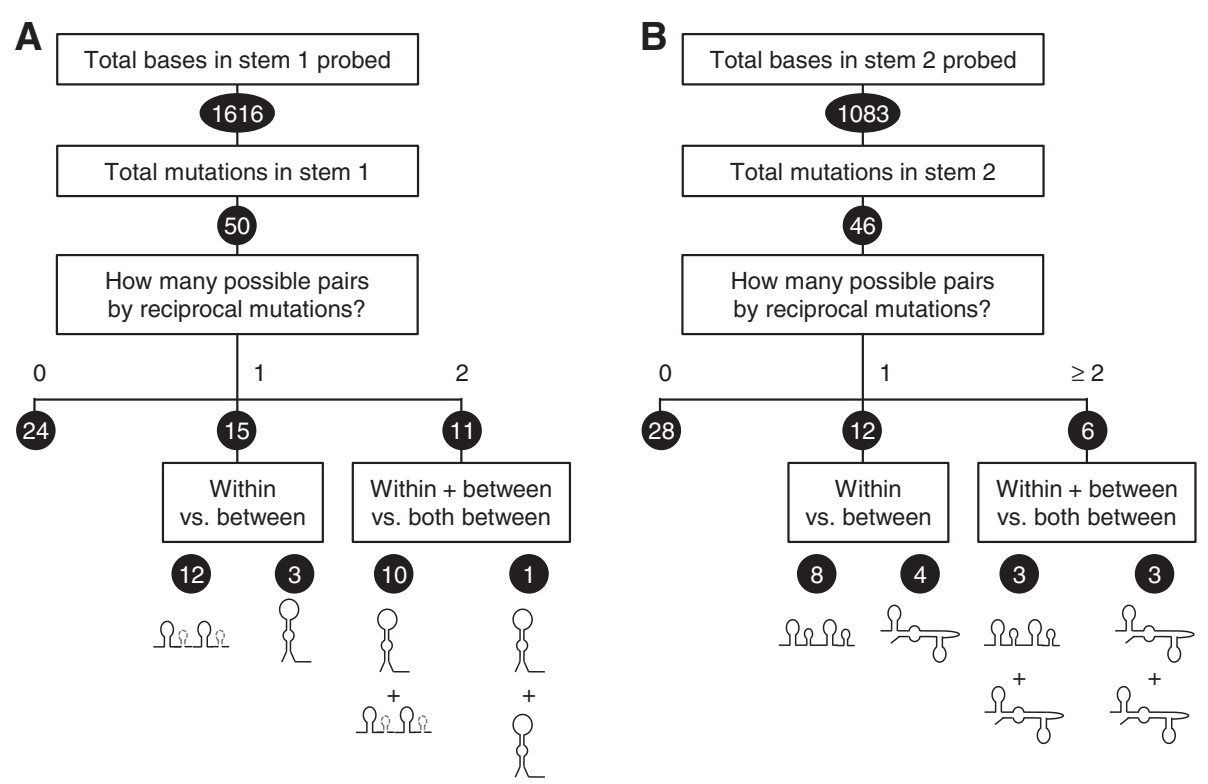

Figure $\mathbf{5}$ Compensatory changes in putative stems of repeat A hairpin sequences of $\mathbf{2 7}$ mammals. (A) All bona fide repeat A core sequences that deviated in sequence from the canonical stem 1 sequence were categorized by their potential to form a base pair with a reciprocally-mutated base within the same repeat $A$ unit or within another unit. (B) As in (A), but stem 2 is analyzed. 
stem-loop 2 formation is less striking than for stem 1 , it is still remarkably high, arguing either that stem 2 indeed forms a stem-loop by pairing within each unit, or that repeat A structure involves a combination of both modes of pairing.

Several secondary structures of repeat A have been proposed based on analysis of repeat A mutants [14], NMR data, [21,22] and RNase footprinting and fluorescence resonance energy transfer data [16]. The first palindrome was suggested to form either a hairpin by pairing within each monomer $[14,21,22]$ or, alternatively, to pair between monomers [16]. Both our targeted mutations in an artificial 2-mer repeat A construct, as well as our assessment of evolutionary sequence conservation, support the intra-repeat pairing model, consistent with outcomes observed in mice [14], that the first palindrome indeed forms a stem to expose the 'ATCG' tetraloop. The mutations we introduced to the second palindrome also resulted in a complete loss of silencing by XIST (Figure 3), supporting the importance of these sequences; however, these mutations did not directly address secondary structure formation. While the second palindrome was proposed to pair within each monomer to form the second stem-loop [14], recent studies suggest that the secondary structure may rather involve pairing between individual repeat A monomers [21,22] or with the T-rich spacers [16]. Our assessment of evolutionary sequence conservation provides evidence in favor of second stem-loop formation, though the frequency of compensatory mutations is less striking than observed for stem-loop 1.

\section{Conclusions}

We utilized a single-copy FRT integration site to generate DOX-inducible XISTcDNA integrations allowing the delineation of the repeat $\mathrm{A}$ monomers as the minimal functional unit that additively contributes to gene silencing. The ability of only two copies of repeat A to reproducibly silence a flanking EGFP reporter gene allowed for further dissection of repeat A sequence to elucidate the relationship between repeat A structure and function. Disruption of either the putative stems or loops of the repeat $A$ abrogated silencing, and mutations of the first palindrome to enforce pairing within a repeat, or between the first and second repeat, supported models that the first palindrome forms a hairpin. An evolutionary analysis of sequence changes within the palindromes allowed assessment of intra- versus inter-repeat pairing in full-length XIST sequences. Again, the model of intrarepeat pairing was favored. The intricate set of events that ultimately lead to X-chromosome inactivation in female mammals remains a vanguard to mammalian epigenetic research. By focusing only on the ability to silence a proximal reporter we have reduced the complexity of deciphering the critical roles of XIST. We demonstrate that a mere $94 \mathrm{bp}$-long sequence of repeat A can silence flanking reporter genes, but not more distal endogenous genes that are silenced by induction of the full-length XIST RNA. Further data on the relationship of repeat A sequence and function will provide a foundation for elucidating the yet unclear connection between the sequence of long non-coding RNAs, like XIST/Xist, and their ability to silence chromatin.

\section{Methods}

\section{Construct generation}

The artificial repeat A construct and its shorter derivatives and mutants were synthesized by GeneArt (now Life Technologies Inc, Burlington, ON, Canada) and cloned into the pcDNA5/FRT/TO plasmid (Life Technologies Inc.) using standard techniques. HT1080 cells were transfected as described previously [13]. Mfold server version 2.3 was used to predict secondary RNA structures (http://mfold.rna.albany.edu) of new constructs.

\section{Cell culture}

Clones harboring single-copy FLP-mediated integrations of XIST constructs into HT1080 fibrosarcoma cell lines were generated and cultured as described previously [13]. The XIST transgenes were induced by doxycycline $(1 \mu \mathrm{g} / \mathrm{mL})$ and cell culture medium was changed every 24 hours.

\section{Identification of the transgene integration site}

Inverse PCR utilizing primers complementary to a sequence within the integrated pEGFP-N1 plasmid (Life Technologies) was used to identify the precise integration site of the XIST - EGFP transgene in the HT1080 23-0.5 + 3\#4 cell line. PCR primer sequences are listed in Additional file 5: Supplementary methods.

\section{qRT-PCR}

RNA was isolated from frozen cell pellets by TRIZOL (Life Technologies Inc.) and treated with DNase I (Roche Diagnostics, Laval, QC Canada) according to the manufacturers' recommendations. Following phenolchloroform extraction, RNA concentration was assessed by spectrophotometry and 0.5 to $2.5 \mu \mathrm{g}$ of RNA was reverse-transcribed by $\mathrm{M}-\mathrm{MLV}$ reverse transcriptase (Invitrogen). Fermentas HS Taq (Thermo Scientific, Waltham, MA USA) and EvaGreen (Biotium Inc., Hayward CA USA) were used in quantitative PCR under the following conditions: 5 minutes $95^{\circ} \mathrm{C}, 40 \mathrm{x}\left(15 \mathrm{sec} .95^{\circ} \mathrm{C}\right.$, 30 sec. $60^{\circ} \mathrm{C}, 60$ sec. $72^{\circ} \mathrm{C}$ ). PCR primer sequences are listed in Additional file 5: Supplementary methods.

\section{Flow cytometry}

HT1080 cell pellets were washed with PBS and resuspended in $0.5 \mathrm{~mL}$ of PBS supplemented with $10 \%$ 
FCS. A total of 30,000 events were recorded using an LSRII flow cytometer (BD Biosciences, Mississauga, ON, Canada). Mean fluorescence intensity of EGFP was assessed by using a combination of $488 \mathrm{~nm}$ laser excitation and a 530/30 $\mathrm{nm}$ bandpass filter.

\section{Allelic discrimination by pyrosequencing}

A total of $2 \mu \mathrm{L}$ of cDNA was added to a standard $25-\mu \mathrm{L}$ pyrosequencing reaction containing $1 \times \mathrm{PCR}$ buffer (QIAGEN, Valencia, CA, USA), $0.2 \mathrm{mM}$ dNTPs, 0.625 unit Hot Start Taq DNA polymerase (QIAGEN), 0.25 $\mu \mathrm{M}$ forward primer and $0.25 \mu \mathrm{M}$ reverse primer. PCR conditions were: $95^{\circ}$ for 15 minutes, 35 cycles of $94^{\circ}$ for $30 \mathrm{sec}, 56.3^{\circ}$ or $58.3^{\circ}$ for $30 \mathrm{sec}$ (see Supplementary table), $72^{\circ}$ for $30 \mathrm{sec}$, and finally $72^{\circ}$ for 10 minutes. Template preparation for pyrosequencing was done according to the manufacturer's protocol, using 10 to 15 $\mu \mathrm{l}$ of PCR products.

\section{Analysis of repeat $A$ core in mammals}

Repeat A sequences in a panel of mammalian species were identified using a combination of BLAST, BLAT and in silico PCR searches of mammalian genomes available through NCBI (http://blast.ncbi.nlm.nih.gov) and ENSEMBL (http:// www.ensembl.org/Multi/blastview) databases and the UCSC genome browser (http://genome.ucsc.edu). A table listing the accession numbers or genomic locations of repeat A sequences is provided in Additional file 5: Supplementary methods. Sequences were aligned using clustalw2 (http:// www.ebi.ac.uk/Tools/msa/clustalw2) and screened to exclude all non-bona fide repeat A CG-rich core sequences from further analyses. CG-rich core sequences that contained bases deviating from the canonical sequence of either stem 1 or stem 2 were identified. Finally, we tested whether such a mutation was reciprocated by a mutation within the same repeat A unit, or in all other repeats of that species.

\section{Additional files}

Additional file 1: Figure S1. ChIP for $\mathrm{H} 3 \mathrm{~K} 27 \mathrm{me} 3$ at silenced promoters. ChIP for H3K27me3 at the EGFP, CLDN16 (2 locations, P1 and P2) and Hyg (2 locations, P1 and P2) promoters that are shown to be silenced by DOX-induced expression of XIST. H3 shows pull-down for all promoters, while IgG shows limited pull-down. MYT1, a silenced gene, is a positive control for H3K27me3 recruitment, and the active APRT gene is used as a negative control.

Additional file 2: Figure S2. Alignment of repeat A sequences in 27 mammals. (A) Sequence alignment of repeat A region in 27 mammalian species. Black circles mark sequences that were not considered bona fide repeat $A$ units and were thus excluded from further analyses. (B) Sequence conservation of 202 core repeat A units among 27 mammalian species. Lines on the $\mathrm{X}$ axis depict (from top to bottom) the position of bases, percent of units that deviate from canonical sequence, the canonical sequence and arrows corresponding to bases forming the hypothesized stem 1 and stem 2 .
Additional file 3: Figure S3. In silico prediction of repeat A mutant structure. Structures and free energies of 2-mer repeat A and mutants created to enforce pairing within each monomer $(A 1, A 2)$ or between the two monomers $(B 1, B 2)$ predicted by mfold. Bases diverging from the canonical repeat A sequence are capitalized and highlighted.

Additional file 4: Figure S4. Analysis of repeat A sequences in 27 mammals. (A) Illustration of the approach used to analyze repeat $A$ sequence alignment data in Figure 5A, B and Additional file 3: Figure S3B, C. (B) Analysis of reciprocal mutations in the stem 1 of individual repeat A units. The table depicts the number of occurrences when mutation in a repeat $A$ unit would allow pairing due to the existence of a reciprocal mutation within the same unit (highlighted), in a different unit or when no reciprocal mutation exists in the species' repeat A (listed in the last column). (C) As in B), but stem 2 is analyzed.

Additional file 5: Supplementary methods. List of accession numbers or sequence coordinates of repeat A sequences used in sequence analyses and table of primer sequences and ChIP methods.

\section{Abbreviations}

ChIP: Chromatin immunoprecipitation; DOX: Doxycycline; EGFP: Enhanced Green Fluorescent Protein gene; ES: Embryonic stem; FCS: Fetal calf serum; Hyg: Hygromycin gene; ncRNA: Non-coding RNA; PBS: Phosphate-buffered saline.

\section{Competing interests}

The authors declare no financial or non-financial competing interests.

\section{Authors' contributions}

JM designed the study, carried out the cloning and molecular genetic studies, and drafted the manuscript. SELB generated the cell lines described and reviewed the manuscript. CY and AMC contributed to the molecular genetic studies and data analysis, and revised the manuscript. CJB participated in the study design and co-ordination, data interpretation and manuscript preparation. All authors read and approved the final manuscript.

\section{Acknowledgements}

This work was funded by a CIHR operating grant (MOP-13690) to CJB.

Received: 6 March 2013 Accepted: 18 June 2013

Published: 1 August 2013

\section{References}

1. Lyon MF: Gene action in the X-chromosome of the mouse (Mus musculus L.). Nature 1961, 190:372-373.

2. Brown CJ, Ballabio A, Rupert JL, Lafreniere RG, Grompe M, Tonlorenzi R, Willard HF: A gene from the region of the human $\mathrm{X}$ inactivation centre is expressed exclusively from the inactive X chromosome. Nature 1991, 349:38-44.

3. Wutz A: Gene silencing in X-chromosome inactivation: advances in understanding facultative heterochromatin formation. Nat Rev Genet 2011, 12:542-553.

4. Brown CJ, Hendrich BD, Rupert JL, Lafreniere RG, Xing Y, Lawrence J, Willard HF: The human XIST gene: analysis of a $17 \mathrm{~kb}$ inactive X-specific RNA that contains conserved repeats and is highly localized within the nucleus. Cell 1992, 71:527-542.

5. Brockdorff N, Ashworth A, Kay GF, McCabe VM, Norris DP, Cooper PJ, Swift $\mathrm{S}$, Rastan $\mathrm{S}$ : The product of the mouse Xist gene is a $15 \mathrm{~kb}$ inactive $\mathrm{X}$ specific transcript containing no conserved ORF and located in the nucleus. Cell 1992, 71:515-526.

6. Duret L, Chureau C, Samain S, Weissenbach J, Avner P: The Xist RNA gene evolved in eutherians by pseudogenization of a protein-coding gene. Science 2006, 312:1653-1655.

7. Elisaphenko EA, Kolesnikov NN, Shevchenko Al, Rogozin IB, Nesterova TB, Brockdorff N, Zakian SM: A dual origin of the Xist gene from a proteincoding gene and a set of transposable elements. PLoS One 2008, 3:e2521.

8. Grant J, Mahadevaiah SK, Khil P, Sangrithi MN, Royo H, Duckworth J, McCarrey JR, VandeBerg JL, Renfree MB, Taylor W, Elgar G, Camerini-Otero RD, Gilchrist MJ, Turner JM: Rsx is a metatherian RNA with Xist-like properties in X-chromosome inactivation. Nature 2012, 487:254-258. 
9. Hoffman LM, Hall L, Batten JL, Young H, Pardasani D, Baetge EE, Lawrence J, Carpenter MK: X-inactivation status varies in human embryonic stem cell lines. Stem Cells 2005, 23:1468-1478.

10. van den Berg IM, Laven JS, Stevens M, Jonkers I, Galjaard RJ, Gribnau J, van Doorninck JH: X chromosome inactivation is initiated in human preimplantation embryos. Am J Hum Genet 2009, 84:771-779.

11. Okamoto I, Patrat C, Thepot D, Peynot N, Fauque P, Daniel N, Diabangouaya $P$, Wolf JP, Renard JP, Duranthon V, Heard E: Eutherian mammals use diverse strategies to initiate $\mathrm{X}$-chromosome inactivation during development. Nature 2011, 472:370-374.

12. Yang C, Chapman AG, Kelsey AD, Minks J, Cotton AM, Brown CJ: Xchromosome inactivation: molecular mechanisms from the human perspective. Hum Genet 2011, 130:175-185.

13. Chow JC, Hall LL, Baldry SE, Thorogood NP, Lawrence JB, Brown CJ: Inducible XIST-dependent X-chromosome inactivation in human somatic cells is reversible. Proc Natl Acad Sci U S A 2007, 104:10104-10109.

14. Wutz A, Rasmussen TP, Jaenisch R: Chromosomal silencing and localization are mediated by different domains of Xist RNA. Nat Genet 2002, 30:167-174.

15. Kanhere A, Viiri K, Araújo CC, Rasaiyaah J, Bouwman RD, Whyte WA, Pereira CF, Brookes E, Walker K, Bell GW, Pombo A, Fisher AG, Young RA, Jenner RG: Short RNAs are transcribed from repressed polycomb target genes and interact with polycomb repressive complex-2. Mol Cell 2010, 38:675-688.

16. Maenner S, Blaud M, Fouillen L, Savoye A, Marchand V, Dubois A, SanglierCianférani S, Van Dorsselaer A, Clerc P, Avner P, Visvikis A, Branlant C: 2-D structure of the A region of Xist RNA and its implication for PRC2 association. PLOS Biol 2010, 8:e1000276.

17. Zhao J, Sun BK, Erwin JA, Song JJ, Lee JT: Polycomb proteins targeted by a short repeat RNA to the mouse $\mathrm{X}$ chromosome. Science 2008, 322:750-756.

18. Hoki Y, Kimura N, Kanbayashi M, Amakawa Y, Ohhata T, Sasaki H, Sado T: A proximal conserved repeat in the Xist gene is essential as a genomic element for X-inactivation in mouse. Development 2009, 136:139-146.

19. Jeon $Y$, Lee JT: YY1 tethers Xist RNA to the inactive $X$ nucleation center. Cell 2011, 146:119-133.

20. Royce-Tolland ME, Andersen AA, Koyfman HR, Talbot DJ, Wutz A, Tonks ID, Kay GF, Panning B: The A-repeat links ASF/SF2-dependent Xist RNA processing with random choice during $\mathrm{X}$ inactivation. Nat Struct Mol Biol 2010, 17:948-954.

21. Duszczyk MM, Zanier K, Sattler M: A NMR strategy to unambiguously distinguish nucleic acid hairpin and duplex conformations applied to a Xist RNA A-repeat. Nucleic Acids Res 2008, 36:7068-7077.

22. Duszczyk MM, Wutz A, Rybin V, Sattler M: The Xist RNA A-repeat comprises a novel AUCG tetraloop fold and a platform for multimerization. RNA 2011, 17:1973-1982.

23. Calabrese JM, Sun W, Song L, Mugford JW, Williams L, Yee D, Starmer J, Mieczkowski P, Crawford GE, Magnuson T: Site-specific silencing of regulatory elements as a mechanism of $\mathrm{X}$ inactivation. Cell 2012, 151:951-963.

24. Wutz $A$, Jaenisch $R$ : A shift from reversible to irreversible $X$ inactivation is triggered during ES cell differentiation. Mol Cell 2000, 5:695-705.

25. Zuker M: Mfold web server for nucleic acid folding and hybridization prediction. Nucleic Acids Res 2003, 31:3406-3415.

doi:10.1186/1756-8935-6-23

Cite this article as: Minks et al: XIST-induced silencing of flanking genes is achieved by additive action of repeat a monomers in human somatic cells. Epigenetics \& Chromatin 2013 6:23.

\section{Submit your next manuscript to BioMed Central and take full advantage of:}

- Convenient online submission

- Thorough peer review

- No space constraints or color figure charges

- Immediate publication on acceptance

- Inclusion in PubMed, CAS, Scopus and Google Scholar

- Research which is freely available for redistribution

Submit your manuscript at www.biomedcentral.com/submit
Ciomed Central 